\title{
Conformational Flexibility in 2,2'-Dioxybiphenyl-chloro-cyclotetraphosphazenes and its Relevance to Polyphosphazene Analogues
}

\author{
Eric W. Ainscough,,${ }^{\star}{ }^{\star}$ Andrew M. Brodie,,${ }^{\star}{ }^{\star}$ Adrian B. Chaplin,,$^{\ddagger}$ Andreas Derwahl,, John A. \\ Harrison $\$$ and Carl A. Otter
}

Chemistry - Institute of Fundamental Sciences, Massey University, Private Bag 11 222, Palmerston North, New Zealand, Institut des Sciences et Ingénierie Chimiques, Ecole Polytechnique Fédérale de Lausanne (EPFL), CH-1015 Lausanne, Switzerland, and Chemistry - Institute of Fundamental Sciences, Massey University, Private Bag 102 904, North Shore Mail Center, Auckland, New Zealand

\section{Electronic Supplementary Material}

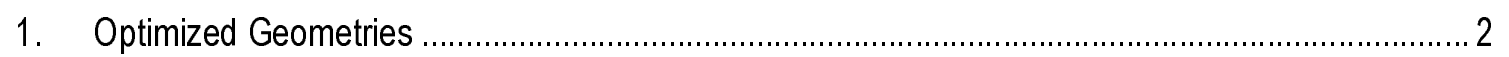

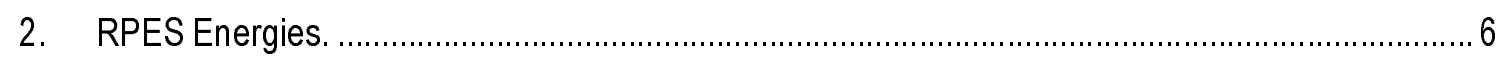

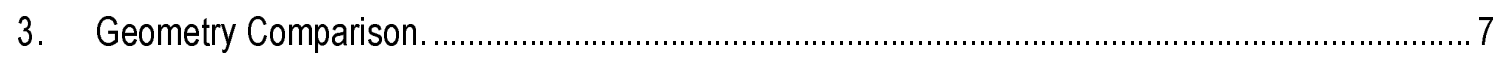




\section{Optimized Geometries}

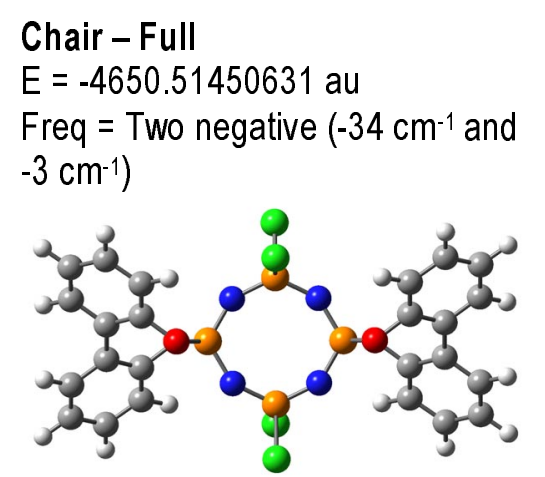

Boat - Full
$E=-4650.51629386$ au

Freq $=$ One negative $\left(-4 \mathrm{~cm}^{-1}\right)$

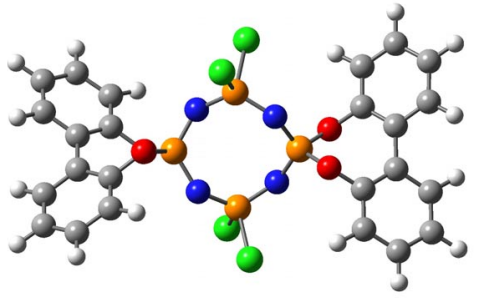

0

1. 150205

1. 898484

1. 150205

0

$-1.150205$

$-1.898484$

$-1.150205$

0.578896

$-0.578896$

0.578896

$-0.578896$

3. 008475

3. 370906

$-3.370906$

$-3.008475$

$-0.720486$

$-1.342903$

$-2.69002$

$-3.459199$

$-2.872485$

$-1.522678$

0.720486

1. 342903

2. 69002

3. 459199

2.872485

1. 522678

$-3.112065$

$-4.512076$

$-3.467845$

$-1.075588$

3. 112065

4.512076

3. 467845

1. 075588

$-0.720486$

$-1.342903$

$-2.69002$

$-3.459199$

$-2.872485$

$-1.522678$

0.720486

1. 342903

2.69002

3. 459199

2. 872485

1. 522678

$-3.112065$

$-4.512076$

$-3.467845$

$-1.075588$

3. 112065

4. 512076

3.467845

1. 075588

0

$-0.41067$

0

1. 548706

$-1.547623$

0.437161

0

$-1.250951$

$-1.135046$

$-0.37882$

$-0.386607$

0.208987

$-1.116955$

$-1.097537$
0

0.753274

0.795324

0.753274

0

$-0.753274$

$-0.795324$

$-0.753274$

$-1.12958$

1.12958

$-1.12958$

1.12958

2. 505193

$-0.675251$

0.675251

$-2.505193$

0.173466

0.7705

1.116864

0.868615

0.276491

$-0.066456$

$-0.173466$

$-0.7705$

$-1.116864$

$-0.868615$

$-0.276491$

0.066456

1.581229

1.134779

0.072532

$-0.548908$

$-1.581229$

$-1.134779$

$-0.072532$

0.548908

0.173466

0.7705

1.116864

0.868615

0.276491

$-0.066456$

$-0.173466$

$-0.7705$

$-1.116864$

$-0.868615$

$-0.276491$

0.066456

1. 581229

1.134779

0.072532

$-0.548908$

$-1.581229$

$-1.134779$

$-0.072532$

0.548908

1. 313992

2. 063806

3. 365865

3. 350076

1. 305028

0

$-0.229024$

$-1.057575$

$-0.636828$

$-1.470056$

$-1.182949$

$-2.655464$

$-3.281172$
2.16085

1. 377802

$-1.377802$

$-2.16085$

1. 377802

0

$-1.377802$

3. 162892

3. 162892

$-3.162892$

$-3.162892$

0

5. 387965

4. 279306

4.279465

5. 414821

6.535124

6.51644

5. 387965

4. 279306

4.279465

5. 414821

6.535124

6.51644

3. 394514

5.420967

7.420316

7. 380645

3. 394514

5.420967

7.420316

7.380645

$-5.387965$

$-4.279306$

$-4.279465$

$-5.414821$

$-6.535124$

$-6.51644$

$-5.387965$

$-4.279306$

$-4.279465$

$-5.414821$

$-6.535124$

$-6.51644$

$-3.394514$

$-5.420967$

$-7.420316$

$-7.380645$

$-3.394514$

$-5.420967$

$-7.420316$

$-7.380645$

2.107678

1. 317064

$-0.004318$

0.383983

$-0.406642$

$-1.314926$

$-2.105335$

3. 10717

4. 22976

5.335925

6.467691

7. 329277

6. 493478

7. 381017 


\begin{tabular}{|c|c|c|c|c|}
\hline & 6 & -1.86147 & -3.041625 & 5.377104 \\
\hline & 1 & -2.431397 & -3.966007 & 5.388518 \\
\hline & 6 & -1.870485 & -2.238285 & 4.238447 \\
\hline & 1 & -2.442622 & -2.50588 & 3.356812 \\
\hline & 8 & -1.195012 & 0.42772 & -3.105611 \\
\hline & 6 & -1.509341 & -0.354581 & -4.224223 \\
\hline & 6 & -0.644975 & -0.365271 & -5.331194 \\
\hline & 6 & -1.065073 & -1.089097 & -6.460119 \\
\hline & 1 & -0.407462 & -1.13597 & -7.323184 \\
\hline & 6 & -2.285075 & -1.760533 & -6.480694 \\
\hline & 1 & -2.578965 & -2.316683 & -7.366096 \\
\hline & 6 & -3.120167 & -1.728113 & -5.361853 \\
\hline & 1 & -4.070973 & -2.25285 & -5.369606 \\
\hline & 6 & -2.729829 & -1.021293 & -4.225974 \\
\hline & 1 & -3.356034 & -0.970084 & -3.341853 \\
\hline & 7 & 0.41067 & -1.313992 & 1.317064 \\
\hline & 15 & 0 & -2.063806 & -0.004318 \\
\hline & 17 & -1.548706 & -3.365865 & 0.383983 \\
\hline & 17 & 1.547623 & -3.350076 & -0.406642 \\
\hline & 7 & -0.437161 & -1.305028 & -1.314926 \\
\hline & 8 & 1.250951 & 0.229024 & 3.10717 \\
\hline & 6 & 1.135046 & 1.057575 & 4.22976 \\
\hline & 6 & 0.37882 & 0.636828 & 5.335925 \\
\hline & 6 & 0.386607 & 1.470056 & 6.467691 \\
\hline & 1 & -0.208987 & 1.182949 & 7.329277 \\
\hline & 6 & 1.116955 & 2.655464 & 6.493478 \\
\hline & 1 & 1.097537 & 3.281172 & 7.381017 \\
\hline & 6 & 1.86147 & 3.041625 & 5.377104 \\
\hline & 1 & 2.431397 & 3.966007 & 5.388518 \\
\hline & 6 & 1.870485 & 2.238285 & 4.238447 \\
\hline & 1 & 2.442622 & 2.50588 & 3.356812 \\
\hline & 8 & 1.195012 & -0.42772 & -3.105611 \\
\hline & 6 & 1.509341 & 0.354581 & -4.224223 \\
\hline & 6 & 0.644975 & 0.365271 & -5.331194 \\
\hline & 6 & 1.065073 & 1.089097 & -6.460119 \\
\hline & 1 & 0.407462 & 1.13597 & -7.323184 \\
\hline & 6 & 2.285075 & 1.760533 & -6.480694 \\
\hline & 1 & 2.578965 & 2.316683 & -7.366096 \\
\hline & 6 & 3.120167 & 1.728113 & -5.361853 \\
\hline & 1 & 4.070973 & 2.25285 & -5.369606 \\
\hline & 6 & 2.729829 & 1.021293 & -4.225974 \\
\hline & 1 & 3.356034 & 0.970084 & -3.341853 \\
\hline Oval - Full & & & & \\
\hline$E=-4650.51564398$ au & $\begin{array}{l}15 \\
7\end{array}$ & $\begin{array}{l}-1.98047 \\
-1.970586\end{array}$ & $\begin{array}{l}0.237264 \\
0.278612\end{array}$ & $\begin{array}{l}-0.712682 \\
0.876701\end{array}$ \\
\hline Frea $=$ All + & 15 & -0.789356 & 0.125253 & 1.915678 \\
\hline & 7 & 0.705601 & 0.281775 & 1.481847 \\
\hline & 17 & -1.132426 & 1.51699 & 3.380062 \\
\hline & 17 & -1.05713 & -1.647645 & 2.936036 \\
\hline & 8 & -2.355442 & 1.684721 & -1.340381 \\
\hline & 8 & -3.262822 & -0.670945 & -1.080761 \\
\hline & 6 & -3.633719 & 2.23193 & -1.182753 \\
\hline & 6 & -3.759724 & 3.367106 & -0.388864 \\
\hline & 1 & -2.884767 & 3.744163 & 0.129706 \\
\hline & 6 & -4.99898 & 3.996359 & -0.287363 \\
\hline & 1 & -5.104505 & 4.881846 & 0.332495 \\
\hline & 6 & -6.097541 & 3.4824 & -0.979537 \\
\hline & 1 & -7.068388 & 3.962359 & -0.897758 \\
\hline & 6 & -5.953946 & 2.344094 & -1.769032 \\
\hline & 1 & -6.815322 & 1.933939 & -2.288014 \\
\hline & 6 & -4.716865 & 1.689412 & -1.893925 \\
\hline & 6 & -4.56942 & 0.495258 & -2.759594 \\
\hline & 6 & -5.191715 & 0.433318 & -4.018036 \\
\hline & 1 & -5.756355 & 1.294529 & -4.36294 \\
\hline & 6 & -5.075739 & -0.690787 & -4.832196 \\
\hline & 1 & -5.563455 & -0.705584 & -5.802516 \\
\hline & 6 & -4.324581 & -1.788321 & -4.406525 \\
\hline & 1 & -4.226491 & -2.666379 & -5.038028 \\
\hline & 6 & -3.693748 & -1.755816 & -3.164265 \\
\hline & 1 & -3.109532 & -2.593339 & -2.798463 \\
\hline & 6 & -3.822742 & -0.626306 & -2.362823 \\
\hline & 15 & 1.98047 & -0.237264 & 0.712682 \\
\hline & 7 & 1.970586 & -0.278612 & -0.876701 \\
\hline & 15 & 0.789356 & -0.125253 & -1.915678 \\
\hline & 7 & -0.705601 & -0.281775 & -1.481847 \\
\hline & 17 & 1.132426 & -1.51699 & -3.380062 \\
\hline & 17 & 1.05713 & 1.647645 & -2.936036 \\
\hline
\end{tabular}


TS (oval/boat) - Full $E=-4650.5152017$ au Freq $=$ One Negative $\left(-16 \mathrm{~cm}^{-1}\right)$

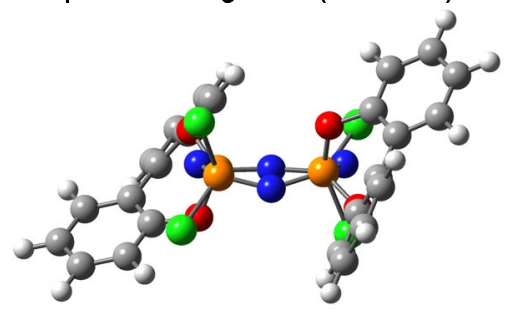

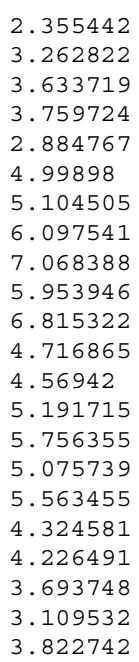

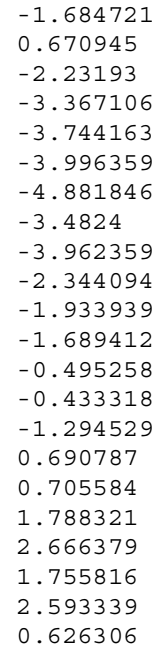




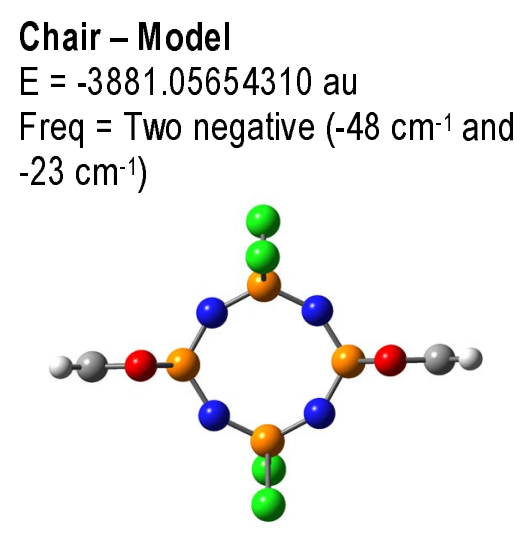

Boat - Model
$E=-3881.05808646$ au

\section{Freq $=$ All +}

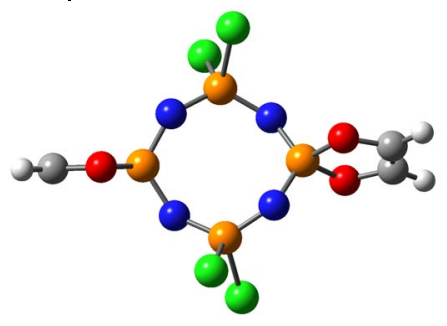

\section{Oval - Model}

$$
E=-3881.05742043 \text { au }
$$

\section{Freq $=$ All +}

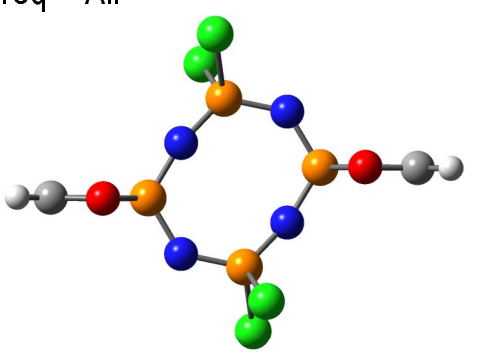

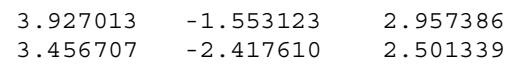

0

0.273155

0

0

$-0.273155$

0

1. 216978

$-1.216978$

1.216978

$-1.216978$

0.665317

0.665317

$-0.665317$

$-0.665317$

1. 369666

$-1.369666$

1. 369666

$-1.369666$

$-0.824821$

2. 209288

$-2.209288$

0.824821

$-0.000112$

1. 305259

2. 061482

3. 339289

3. 342595

1. 305694

0.000112

0. 298235

$-0.298235$

$-1.305694$

$-2.061482$

$-3.342595$

$-3.339289$

$-1.305259$

$-0.298235$

0.298235

0.164111

$-0.164302$

$-0.164111$

0.164302

$-0.339152$

0.339467

$-0.339467$

0.339152

$-1.154262$

$-1.142762$

$-0.575495$

0.376255

$-2.162512$

0.366196

1. 154262

$-2.704035$

0.728667

$-0.366196$

0.575495

$-0.376255$

2. 162512

1. 142762

$-0.728667$

2. 704035

2. 920183

1. 840213

$-1.840213$

$-2.920183$

$-1.691355$

$-3.914304$

1. 691355

3. 914304
0

1. 364802

.063749

$1.364802-1.380376$

$0-2.169024$

$-1.364802 \quad 1.380376$

$-2.063749 \quad 0$

$-1.364802 \quad-1.380376$

$-0.03629 \quad 3.271678$

$0.03629 \quad 3.271678$

$-0.03629-3.271678$

$0.03629-3.271678$

$-0.01907 \quad 4.552195$

$-0.01907-4.552195$

$0.01907-4.552195$

$0.01907 \quad 4.552195$

$-0.038591-5.368701$

$0.038591 \quad-5.368701$

$\begin{array}{ll}-0.038591 & 5.368701\end{array}$

$0.038591 \quad 5.368701$

3.782659

2.76814

$-2.76814$

$-3.782659$

2.120629

1. 323893

0.0001

$-0.391021$

0.390873

$-1.323432$

$-2.120629$

3. 220453

$-3.220453$

1.323432

$-0.0001$

$-0.390873$

0.391021

$-1.323893$

3.222723

$-3.222723$

$-4.503027$

$-4.50179$

4. 503027

4.50179

5.32026

5.317714

$-5.317714$

$-5.32026$

$-1.157064$

$-1.163419$

$-1.15676$

$-2.943512$

$-1.2286$

0.007969

1. 157064

$-1.201764$

2. 622119

$-0.007969$

1.15676

2. 943512

1. 2286

1.163419

$-2.622119$

1. 201764

2. 41242

3. 189656

$-3.189656$

$-2.41242$

$-4.16622$

$-2.565507$

4.16622

2. 565507
0

0

$-0.000797$

0.409948

0.000822

1. 553741

$-1.549433$

$-0.409985$

$-0.000797$

$-1.183312$

$-0.409985$

0.000822

$-1.549433$

1. 553741

0.409948

1. 17965

1.17965

0.641923

$-0.647997$

0.641923

$-0.647997$

1. 323581

$-1.331191$

$-1.331191$

1. 323581

$-1.391708$

0.177129

1.63

2. 000979

2. 915934

2. 148753

1. 391708

$-1.929086$

2.006802

$-1.63$

$-2.000979$

$-2.915934$

$-0.177129$

$-2.006802$

1. 929086

2. 586543

2. 628714

$-2.628714$

$-2.586543$

$-3.06172$

$-2.975655$

3. 06172

2. 975655
$-1.183312$

$-2.148753$ 
TS (oval/boat) - Model

Z.P.C. $E=-3881.05741026 \mathrm{au}$

Freq $=$ One negative $\left(-12 \mathrm{~cm}^{-1}\right)$

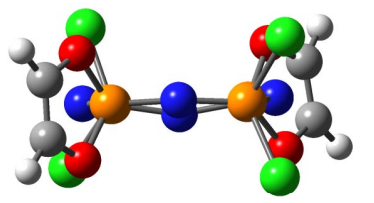

$$
\begin{aligned}
& -2.055676 \\
& -1.396095 \\
& -0.461999 \\
& 1.111743 \\
& 2.068218 \\
& -1.112986 \\
& 0.461813 \\
& 1.42517 \\
& -0.834891 \\
& -1.072774 \\
& 0.934417 \\
& 0.929121 \\
& -3.203626 \\
& -3.097887 \\
& 3.161937 \\
& 3.165136 \\
& -4.453958 \\
& 4.436349 \\
& 4.438116 \\
& -4.396711 \\
& 5.247288 \\
& 5.250565 \\
& -5.296418 \\
& -5.178694
\end{aligned}
$$

\section{RPES Energies.}

Table S1: RPES Energies

\begin{tabular}{cc}
\hline CI(axial)-P.P.Cl(axial) dihedral angle $\mathcal{I}^{\circ}$ & Energy / Hartrees \\
\hline-17.5 & -3881.053905 \\
-15 & -3881.054588 \\
-12.5 & -3881.055146 \\
-10 & -3881.05565 \\
-7.5 & -3881.056041 \\
-5 & -3881.05632 \\
-2.5 & -3881.056487 \\
0 & -3881.056543 \\
2.5 & -3881.056487 \\
5 & -3881.05632 \\
7.5 & -3881.056041 \\
10 & -3881.05565 \\
12.5 & -3881.055146 \\
15 & -3881.054588 \\
17.5 & -3881.053905 \\
\hline
\end{tabular}




\section{Geometry Comparison.}

Table S2: Selected Distances /Å

\begin{tabular}{|c|c|c|c|c|c|c|c|c|c|c|c|c|}
\hline & $P N$ & Expt. & Full & Model & $P O$ & Expt. & Full & Model & $\mathrm{PCl}$ & Expt. & Full & Model \\
\hline \multirow[t]{4}{*}{$2 A a$} & P1N1A & $1.546(14)$ & 1.582 & 1.576 & P101 & $1.582(2)$ & 1.617 & 1.643 & P2ACl1A & $2.042(4)$ & 2.081 & 2.060 \\
\hline & P2AN1A & $1.59(2)$ & 1.568 & 1.571 & P3O2 & $1.581(2)$ & 1.617 & 1.643 & $\mathrm{P} 2 \mathrm{ACl} 2 \mathrm{~A}$ & $1.994(2)$ & 2.039 & 2.040 \\
\hline & P2AN2A & $1.59(2)$ & 1.568 & 1.571 & & & & & & & & \\
\hline & P3N2A & $1.57(2)$ & 1.582 & 1.576 & & & & & & & & \\
\hline \multirow[t]{4}{*}{$2 A C$} & P1N1C & $1.71(3)$ & 1.587 & 1.584 & P101 & $1.582(2)$ & 1.617 & 1.642 & $\mathrm{P} 2 \mathrm{CCl} 1 \mathrm{C}$ & $2.079(6)$ & 2.060 & 2.049 \\
\hline & $\mathrm{P} 2 \mathrm{CN} 1 \mathrm{C}$ & $1.60(4)$ & 1.576 & 1.579 & $\mathrm{P} 3 \mathrm{O} 2$ & $1.581(2)$ & 1.617 & 1.642 & $\mathrm{P} 2 \mathrm{CCl} 2 \mathrm{C}$ & $1.954(6)$ & 2.052 & 2.049 \\
\hline & $\mathrm{P} 2 \mathrm{CN} 2 \mathrm{C}$ & $1.49(4)$ & 1.574 & 1.579 & & & & & & & & \\
\hline & P3N2C & $1.63(4)$ & 1.588 & 1.584 & & & & & & & & \\
\hline \multirow[t]{4}{*}{$2 B$} & $\mathrm{P} 1 \mathrm{~N} 1$ & $1.571(3)$ & 1.590 & 1.586 & P101 & $1.577(3)$ & 1.614 & 1.645 & $\mathrm{P} 2 \mathrm{Cl} 1$ & $1.9880(12)$ & 2.049 & 2.044 \\
\hline & P1N2\# & $1.548(3)$ & 1.577 & 1.569 & P102 & $1.583(2)$ & 1.622 & 1.641 & $\mathrm{P} 2 \mathrm{Cl} 2$ & $1.9885(13)$ & 2.063 & 2.058 \\
\hline & P2N1 & $1.558(3)$ & 1.581 & 1.585 & & & & & & & & \\
\hline & P2N2 & $1.516(3)$ & 1.564 & 1.560 & & & & & & & & \\
\hline
\end{tabular}

Table S3: Selected Angles $/^{\circ}$

\begin{tabular}{lllllllll}
\hline & PNP & Expt & Full & Model & NPN & Expt & Full & Model \\
\hline 2Aa & P1N1AP2A & $132.6(10)$ & 142.6 & 145.6 & N1AP1N1A\# & $117(2)$ & 120.7 & 120.0 \\
& P2AN2AP3 & $128(2)$ & 142.6 & 145.6 & N1AP2AN2A & $117.2(10)$ & 122.9 & 122.9 \\
& & & & & N2AP3N2A\# & $120.6(14)$ & 120.7 & 120.0 \\
$2 A C$ & P1N1CP2C & $126(2)$ & 137.2 & 138.6 & N1P1N1C\# & $124(2)$ & 120.3 & 119.6 \\
& P2CN2CP3 & $142(3)$ & 138.2 & 138.6 & N1P2CN2C & $125(2)$ & 122.8 & 122.8 \\
& & & & & N2CP3N2C\# & $117(2)$ & 120.3 & 119.6 \\
$2 B$ & P1N1P2 & $131.1(2)$ & 131.2 & 132.2 & N1P1N2\# & $119.3(2)$ & 119.4 & 118.5 \\
& P2N2P1A & $163.7(2)$ & 151.0 & 159.1 & N1P2N2 & $123.1(2)$ & 121.5 & 121.6 \\
\hline
\end{tabular}

